\title{
Efficient design of Automatic Train Operation speed profiles with on board energy storage devices
}

\author{
M. Domínguez ${ }^{1}$, A. Fernández ${ }^{1}$, A. P. Cucala ${ }^{1}$ \& J. Blanquer ${ }^{2}$ \\ ${ }^{I}$ Instituto de Investigación Tecnológica, \\ Escuela Técnica Superior de Ingeniería (ICAI), \\ Universidad Pontificia Comillas, Spain \\ ${ }^{2}$ Metro de Madrid, Spain
}

\begin{abstract}
Energy usage in electrical railway systems is being studied in order to find technologies and developments for increasing energy efficiency. It is not only an environmental problem, but also concerns railway infrastructures as an economical aspect. The problem is finding which system to invest in for decreasing energy consumption and costs. In this paper, two possibilities are studied. The first one is the redesign of the ATO (Automatic Train Operation) speed profiles of metro lines. The speed commands in service nowadays were selected based on time and comfort criteria. In addition, in this paper the consideration of energetic criteria is taken into account. Complementing the previous possibility, the implementation of an on board energy storage device is evaluated. The regenerated energy of electrical brakes in metropolitan railways is not used if there is no other train starting up at the same time, and it is wasted with heating resistors. With the aim of taking advantage of regenerative energy, the economical and energetic advantages of investing in an on board storage device, despite its additional mass, are studied. Both approaches are finally jointed, obtaining speed profiles that are even more efficient with the implementation of the device. Optimal Pareto curves, where the best solutions are placed, are modified taking into account on board storage devices. A simulator is needed for the proper simulation of all the possible speed commands. It has been developed and validated with measurements in line 10 of the Madrid Underground. Solutions show that about $25 \%$ of energy savings are expected with only the speed profiles redesign. In addition, it is shown how these
\end{abstract}


optimal speed profiles are modified when an on board energy storage device is also taken into account.

Keywords: regenerative energy, ecodriving, electrical brake, metropolitan trains, ATO speed profiles, optimal design.

\section{Introduction}

Energy efficiency in railway systems is nowadays a key topic being studied in order to reduce energy consumption and costs. With this end in view, different technologies, developments or strategies are being researched, and tested from the point of view of driving optimization, capacity and optimal use of regenerative braking.

In order to find speed profiles which optimise energy use, mathematical models have been applied to principally optimal control techniques. In [1] the optimal speed profile it is calculated with the maximum principle. The study in [2] considers the problem of the optimal driving strategy based on a generalised equation of motion that can be used in discrete and continuous control. The result is a theoretical approach to the search for the switching points of the driving mode. The authors of [3], seeing the difficulties of resolving the optimal control problem with numerical techniques, developed a discrete dynamic programming algorithm. They use kinetic energy instead of speed and obtain an analytical solution in real time. In [4] Bellman's dynamic programming has also been used to optimise the running profile of a train. The authors transform the original problem into a multistage decision process accomplished by linearization and time-uniform discretization. These approaches include simplifications in their track, trains and driving models. This means that they are not appropriate for the optimal design of Metro ATO speed profiles given the short inter-stations in metropolitan lines, and the differences of a few seconds between the ATO profiles to be designed. Therefore, accurate models are needed.

The difficulty involved in the analytical resolution of the problem means that approaches based on simulation are an alternative. They do not require simplifications and enable an accurate calculation of running times and energy consumption, as [5] manual driving modelling for freight trains. A number of optimisation techniques have been used in combination with simulation. In [6], genetic algorithms (GA) are used. A fitness function with variable weightings was used to identify optimal train trajectories. The influence of the weightings is clear. Artificial Neural Networks have also been used. In [7], it is proposed that they are used to obtain the optimal coasting speed. The objective function is formulated by considering the cost of energy consumption and the cost of passenger travelling time. In [8], Chang et al. include Pareto efficiency in differential evolution to find a trade-off between punctuality, consumption and comfort. However, these models cannot be applied to the realistic case of the Madrid Underground. The features of the ATO system considered (see [9]) make necessary a different approach which, rather than using a continuous control curve, optimises the discrete configuration parameters of the equipment and takes also into account operative and comfort restrictions and the highly irregular track gradients. 
With respect to regenerative braking, different approaches are also found. The regenerated energy of electrical brakes in metropolitan railways is not used if there is no other train starting up at the same time in the same electrical section, or there are not inverting substations, with the result that the energy is wasted heating resistors banks. This means that it is needed to equip the system with devices which allow storing energy in the train or at substations [13]. These devices can be supercapacitors, flywheels and SMES (Superconducting magnetic energy storage). Their advantages against regeneration between trains, is that it is not needed another train available to use the regenerated energy [10]. Moreover they can be used for voltage regulation [11] and reducing energy demand without having an effect in transport efficiency and punctuality [12]. The storage devices at substations require energy to be transferred using the lines, which leads to transmission loses. So, it is avoided by placing the device on-board vehicles [14].

This paper takes and combines two of those strategies. First of all, the redesign of the ATO speed profiles of a line of the Madrid Underground has been carried out. In the Madrid Underground, trains are operated according to the speed commands they receive from balises. These commands define a particular speed profile and running time, with associated energy usage (consumption). The design of speed profiles usually takes into account running times and comfort criteria, but not energy consumption criteria. In this paper, a computer aided procedure for the selection of optimal speed profiles, including energy consumption, which does not have an effect on running times is presented. It is a continuation of the work in [9]. To this end, the equations and algorithms that define the train motion and ATO control have been modelled and implemented in a very detailed simulator. This simulator includes an automatic generator of every possible profile and a graphical assistant for the selection of speed commands in accordance with decision theory techniques. It has been developed and validated with measurements in line 10 of the Madrid Underground.

Secondly, it is evaluated the implementation of an on board energy storage device analyzing the advantages in a new design in which the regenerated energy can be stored and feed the train, without forgetting the additional mass of this device. With that new design, speed profiles even more efficient with the consideration of the on board storage device, are obtained. Some authors have suggested to optimize the charge/discharge of the energy storage devices and speed profiles together [15]. With the aim of developing a realistic study, in this paper it is the train and the speed profile he follows what leads the operation of the storage device. Thus, a redesign has been carried out obtaining new modified optimal Pareto curves, where the best solutions are placed.

\section{Models and simulator}

\subsection{Operation}

When designing an energy efficient driving pattern, the decision variables are running time and energy consumption whilst the comfort criteria must be met. The proposed design method is based on the accurate simulation of all the 


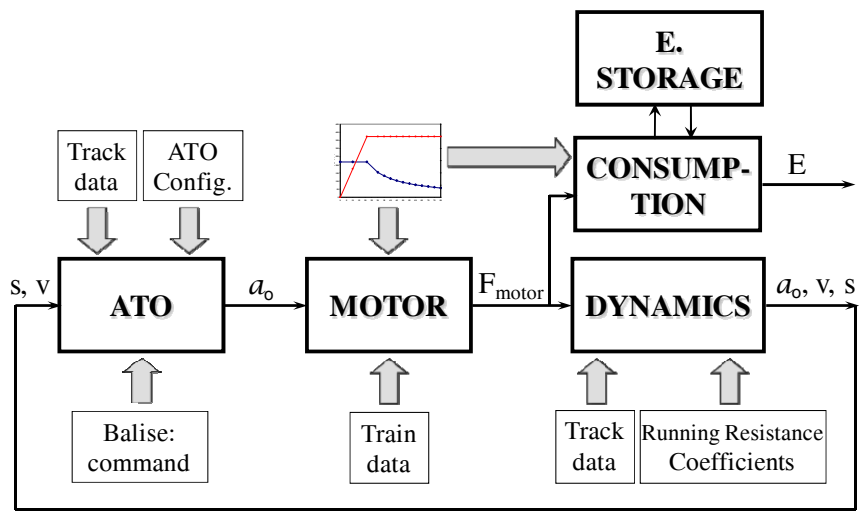

Figure 1: $\quad$ Block diagram of the modularized model simulator.

possible combinations of ATO speed commands for each inter-station in order to obtain precise results of these variables. To achieve this accuracy, the simulation model has been modularized. Each module represents the different subsystems of a real train (Figure 1).

The simulator is composed of five modules: ATO equipment simulator, motor, train dynamics model and train consumption model as well as the model of an on board energy storage device. This modular architecture allows the validation of each module separately and an easy adjustment for specific features of a particular ATO equipment. To this end, the simulator input interfaces are designed to enable the definition of track layout, train characteristics, and ATO system configuration.

The ATO model represents the control logic of the driving. At each simulation step, the position and speed of the train is inputted there. Then, an acceleration set value is calculated depending on the state of the train: motoring, braking to target speed, braking to stop etc. This value is sent to the motor module which translates it as the ratio between required force and maximum traction force corresponding to the speed at each simulation step. Motor needs the mass of the train plus the rotational inertial effect and the traction effort available depending on the speed to calculate the force needed to follow the acceleration set value of the ATO module. Then, a jerk limitation checks there are no abrupt changes in force in transitions like traction-braking or brakingtraction in order to assure the comfort of passengers. Subsequently, the new acceleration, speed and position of the train must be calculated. For that purpose the resistance to train movement is needed. The track gradient resistance $F_{g}$ is the resistive force due to gravity, positive for ramps and negative for slopes and it is calculated from a list with the initial and final points of downhill and uphill sections, their values, and the slope transition curves. Curves are treated as equivalent slopes added to the actual ones. At each simulation step, an average of the gradient where the train is situated is calculated.

Finally, the energy consumption $E$ is recalculated according to the time increment $\Delta t$ and the current $I$ at each simulation step. A constant line voltage $U$ 
is assumed. The current corresponding to the maximum force is the only one known. The consumed one could be calculated, assuming a constant efficiency, but this assumption would not be realistic. Therefore, a model including the variation of the efficiency depending on the ratio between the required and the maximum force is needed.

Train velocity, acceleration, traction or brake force and energy consumption are computed at each simulation step and they would be the input data for the next simulation step.

\subsection{Measurements}

In order to record real data of trains in Line 10 of the Madrid Underground, a laptop was connected on board to the Traction Control System while trains were travelling with flat-out. These measurements have been used to adjust the simulator and validate some data. For example, the differences between the theoretical motor curves and the real ones (measured) have been found and they are shown in Figure 2. The empirical curves are now used instead of the provided one.

A comparison of complete simulations and measured data of running times and energy consumption was also carried out in order to validate the simulator. An average difference of $7.7 \%$ in energy usage and $1.4 \%$ in running times is obtained.

\subsection{Simulations}

The simulator combines all the possible commands that the ATO system provides. Thus, all the possible speed profiles for each inter-station are obtained. The solution space is plotted in a time-consumption graph with every profile characterized by its running time and consumption. Moreover, the simulator indicates which profiles are not available to be implemented because of comfort or operational restrictions. An example is given in Figure 3.

In the Madrid Underground, four alternative speed profiles per inter-station need to be programmed in the Traffic Regulation System. This set of profiles has increasing running times from the first (flat out, the fastest) to the fourth (slowest). If the optimal profiles are chosen, they will also have decreasing
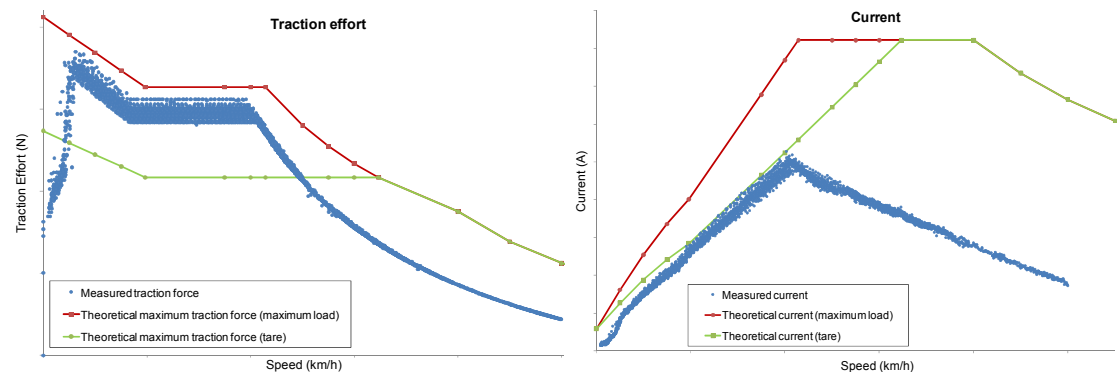

Figure 2: $\quad$ Experimental and theoretical motor curves. 


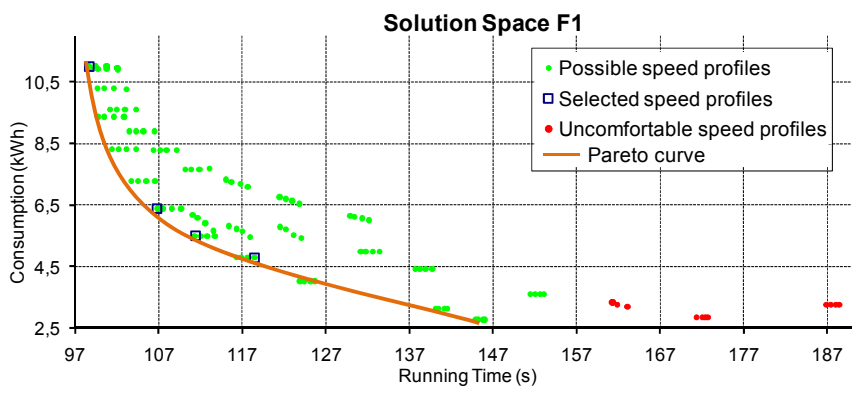

Figure 3: $\quad$ Solution space of Fuencarral platform 1 with uncomfortable speed profiles.

energy consumption according to the shape of the Pareto curve as it is shown in Figure 3. Consequently, this is a multicriteria problem where the aim is to find an appropriate trade-off between energy consumption and running times. Decision theory techniques have been used to solve it.

The proposed procedure follows three criteria: domination, sensitivity and uniform distribution of running times. The following description of the procedure will be illustrated with a realistic application of the Madrid Underground. The energy consumption and running times of the current speed profiles and the proposed ones will be compared in order to value the achievable energy saving.

\section{Case study}

The procedure has been applied to the redesign of all the ATO speed profiles of Line 10 of the Madrid Underground. Some considerations must be taken into account:

- Four speed profiles per inter-station are looked for.

- The selected speed profiles must be comfortable.

- The first profile is the flat out.

- The maximum running time gap between the fastest and the slowest speed profile is limited in practice, so the slowest profile must be moved and placed before the flat slope of the Pareto curve if it is necessary to observe this restriction.

The advantages obtained with the redesign are:

- A temporal uniform distribution of the four speed profiles for each interstation. An example is given in Figure 4 where proposed design and current one are compared. The speed profiles 3 and 4 of the current set consume the same energy with different running times. It takes the second and third profile almost the same time to travel the inter-station and the flat area of the Pareto curve is hardly well-spent. In contrast, the new design proposes profiles over the Pareto curve with a similar gap time between them which favours a proper operation of the traffic regulation system. 
- Comfort. The example in Figure 5 is clear. The speed profile in service nowadays in Lago platform 1 is quite uncomfortable because of the consecutive periods of coasting and motoring. The simulator allows verifying the comfort of the selected profiles.

- An important energy saving without almost affecting running times is achieved. Figure 6 shows how up to $35.1 \%$ savings is reached in Santiago Bernabéu platform 1 maintaining the running time between stations.

The simulation results show that as an average $20.6 \%$ of savings are expected with the sets of profiles redesign being even $25.0 \%$ with the newly designed speed profile number 4 (see Table 1). These results are achieved with only a $2.6 \%$ of running times modification. Although the results are based on simulations yet, it is a reliable value since the simulator has been validated with real measurements.

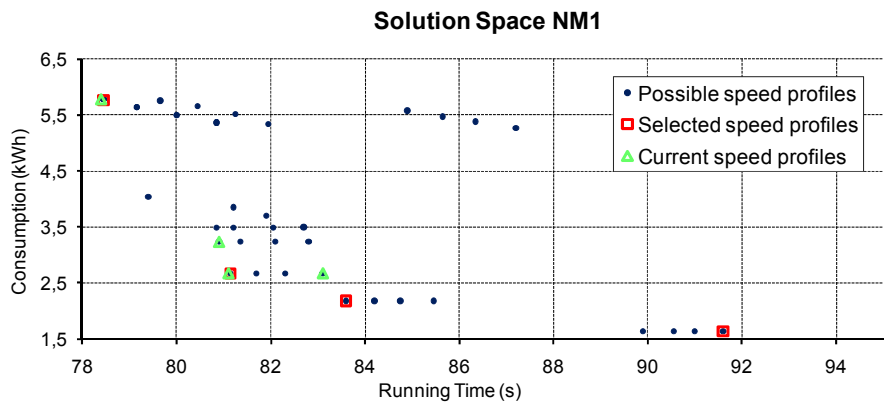

Figure 4: Temporal uniform distribution of the proposed speed profiles in contrast with the current design.

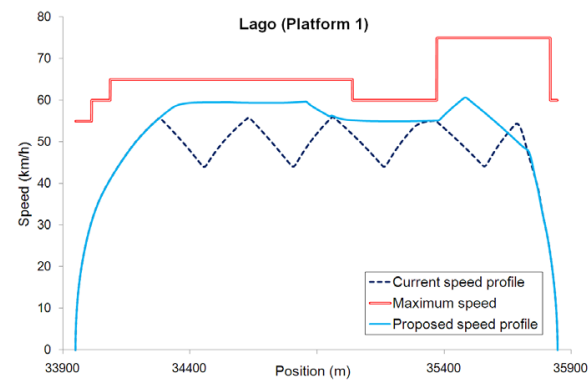

Figure 5: $\quad$ Proposed speed profile instead of the uncomfortable current one. 
Solution Space SB1

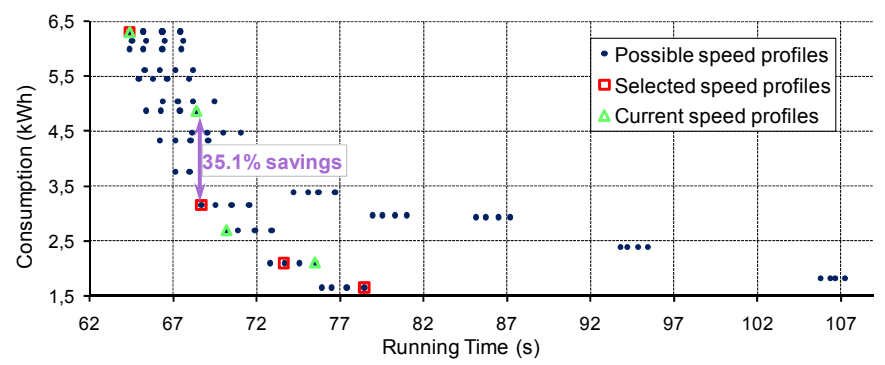

Figure 6: Energy saving achieved with the newly designed profiles.

\section{Consideration of an on board energy storage device}

As shown before, with a proper design of the ATO speed profiles it is possible to achieve important savings due to the selection of the optimal strategies of speed holding or coasting-motoring which decreases the traction consumption. Too furthermore, taking into account the advantages of the regenerative brake, the total energy consumption of the train could decrease considerably. One of the possible technologies for a well spending of this energy is the use of on board energy storage devices. Therefore, a study of the convenience of the implementation in trains of these devices has been carried out. Trains could store their own regenerated energy while braking and use it during the next starting. In order to evaluate the convenience of using them, the previous design has been carried out again considering on-board storage devices, so optimal curves are modified.

With the aim of obtaining realistic results, an on board device with actual features has been look up on the bibliography. The selected technology to be incorporated is "MITRAC Energy Saver" of Bombardier [16]. It is working from September 2003 in a LRV on Mannheim. It is composed by 640 UltraCaps with a capacity of $1800 \mathrm{~F}$ each. Its mass is $477 \mathrm{~kg}$ with a maximum power of $300 \mathrm{~kW}$. Simulations have also been carried out in a European metropolitan system in an 8 vehicles train and a tare of $165 \mathrm{t}$ with 6 devices of $1.5 \mathrm{kWh}$ each [17]. In the present study 4 devices have been assumed with a total mass of $\mathrm{M}=477 \mathrm{x} 4=1905 \mathrm{~kg}$, a maximum power of $300 \mathrm{~kW}$ and $4 \times 1.5=6 \mathrm{kWh}$ possible energy to storage. Moreover, 95\% efficiency has been assumed [18].

\subsection{Particular cases}

Before showing the obtained results, two particular (but real) cases are going to be detailed. One of them is an inter-station situated in an uphill section (Lago platform 1) and the other one in a downhill section (Batán platform 2). Both, the two flat out speed profiles as well as the track gradient are shown in Figure 7.

The redesigns have been carried out taking into account an on board energy storage device which is $50 \%$ charged before travelling an inter-station. In Figure 8 the solution space of the uphill inter-station is shown. It is possible to see the 


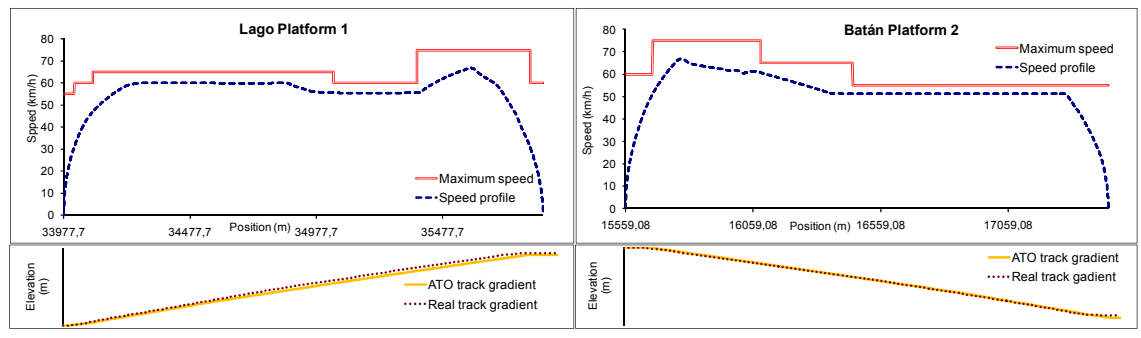

Figure 7: Particular cases.

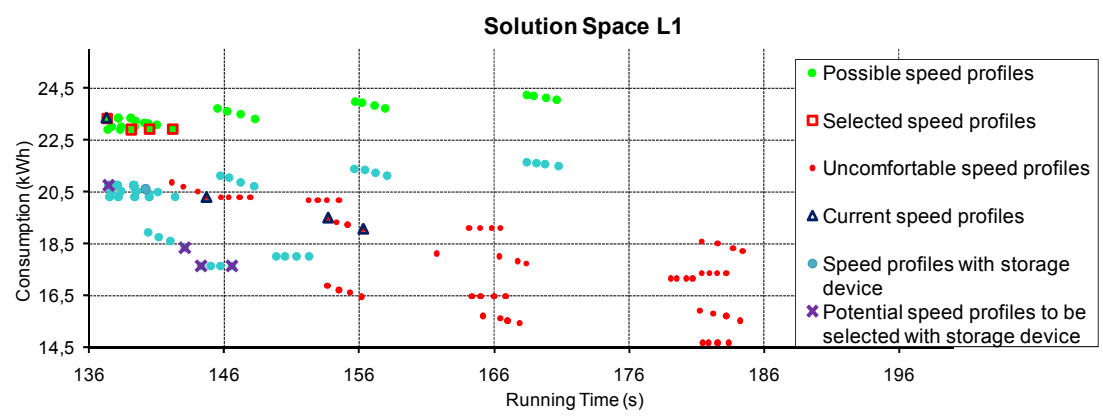

\begin{tabular}{|c|c|c|c|c|c|}
\hline \multicolumn{2}{|c|}{ Design without storage } & \multicolumn{2}{c|}{ Design with storage } & \multicolumn{2}{c|}{ Differences } \\
\hline R. Time (s) & E.Consumption (kWh) & R. Time (s) & E.Consumption (kWh) & R. Time (\%) & E.Consumption (\%) \\
\hline 137.3 & 23.32 & 137.4 & 20.75 & -0.07 & 11.05 \\
\hline 139.1 & 22.89 & 144.3 & 17.64 & -3.70 & 22.95 \\
\hline 140.5 & 22.92 & 143.1 & 18.33 & -1.85 & 20.00 \\
\hline 142.2 & 22.92 & 146.6 & 17.64 & -3.09 & 23.03 \\
\hline
\end{tabular}

Figure 8: $\quad$ Possible design in Lago platform 1.

comparison between the design previously done without storage and the current one. Speed profiles characteristics are shown in the table as well as the energy saving expected: up to $23 \%$ with the fourth profile. A comparison with the profiles currently in service is not possible because they are not observing the comfort restrictions defined for the new design.

Being the inter-station on a downhill section, the potential saving is higher because of the necessity of using electrical braking. An example is given in Figure 9 where almost a $50 \%$ of saving would be possible to achieve with the second profile. Doing the comparison with the profile number 2 currently in service, the consumption could decrease almost $70 \%$.

It is important to notice that in both cases the speed commands which lead to the selected profiles, change when a storage device is considered. That is to say that the optimal profiles are moved not only in " $y$ " axis but also could have a different running time (besides the additional time that the mass storage can mean). On the contrary, there are inter-stations where the optimal Pareto curve is just moved in consumption as the example in Figure 10. 


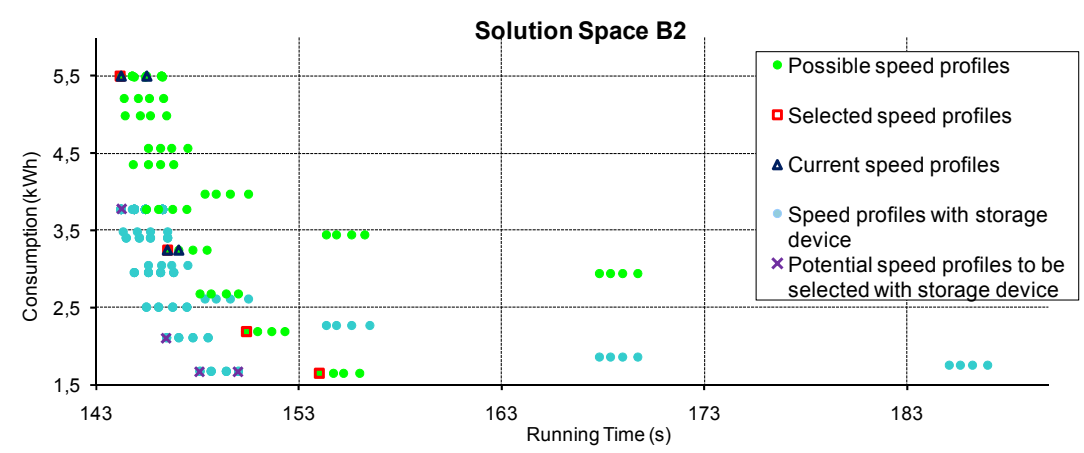

\begin{tabular}{|c|c|c|c|c|c|}
\hline \multicolumn{2}{|c|}{ Design without storage } & \multicolumn{2}{c|}{ Design with storage } & \multicolumn{2}{c|}{ Differences } \\
\hline R. Time (s) & E.Consumption (kWh) & R. Time (s) & E.Consumption (kWh) & R. Time (\%) & E.Consumption (\%) \\
\hline 144.2 & 5.50 & 144.2 & 3.77 & -0.03 & 31.36 \\
\hline 146.5 & 3.24 & 148.1 & 1.67 & -1.09 & 48.52 \\
\hline 150.4 & 2.18 & 150.9 & 1.29 & -0.33 & 40.85 \\
\hline 154.0 & 1.64 & 154.0 & 0.88 & 0.00 & 46.34 \\
\hline
\end{tabular}

Figure 9: $\quad$ Possible design in Batán platform 2.

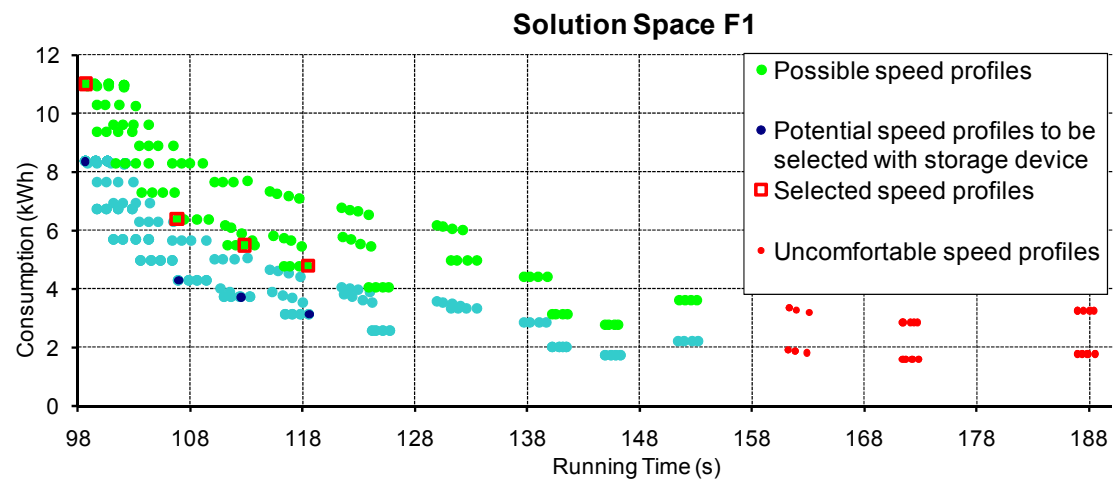

Figure 10: $\quad$ Possible design in Fuencarral platform 1.

\section{Results}

Table 1 shows average results of savings. With the implementation of an on board 50\% charged storage device, energy consumption would decrease up to $40 \%$ regarding to the previous design proposed. It would be $47.5 \%$ of savings regarding to the current situation, that is to say, to the speed profiles in service nowadays in Line 10 of the Madrid Underground. Moreover, the additional mass of the device only increases the running time of the flat out in $0.03 \%$. 
Table 1: $\quad$ Summary table. Average energy savings with the proposed designs.

\begin{tabular}{|l|c|c|c|c|c|c|}
\cline { 2 - 7 } & \multicolumn{2}{c|}{$\begin{array}{c}\text { Design without storage } \\
\text { regarding to current design }\end{array}$} & $\begin{array}{c}\text { Design with storage regarding } \\
\text { to design without storage }\end{array}$ & \multicolumn{2}{c|}{$\begin{array}{c}\text { Design with storage } \\
\text { regarding to current design }\end{array}$} \\
\cline { 2 - 7 } & R. Time (\%) & E. saving (\%) & R. Time (\%) & E. saving (\%) & R. Time (\%) & E. saving (\%) \\
\hline Profile 2 & -0.49 & 16.38 & 0.08 & 32.29 & -0.41 & 43.38 \\
\hline Profile 3 & -2.24 & 20.38 & -0.07 & 33.69 & -2.32 & 47.21 \\
\hline Profile 4 & -5.19 & 25.05 & 0.05 & 35.85 & -5.14 & 51.92 \\
\hline & $-\mathbf{2 . 6 4}$ & $\mathbf{2 0 . 6 0}$ & $\mathbf{0 . 0 2}$ & $\mathbf{3 3 . 9 5}$ & $-\mathbf{2 . 6 2}$ & $\mathbf{4 7 . 5 0}$ \\
\hline
\end{tabular}

\section{Conclusions}

A detailed simulator of the particular ATO system of the Madrid Underground has been developed in order to obtain a realistic simulation that allows calculating slight differences between alternative speed profiles. In the case study of Madrid Underground, these differences can be a few seconds. Thanks to that, it has been possible to carry out a realistic design of the speed profiles of Line 10 . The newly designed profiles against the speed profiles currently being used result in $20 \%$ of savings as an average.

Too furthermore, taking into account the implementation of an on board storage device, up to $47.5 \%$ of savings could be expected regarding to the currently speed profiles. The design has been carried out with an initial charge of $50 \%$ supposed. It would be possible with negligible increase in the running times of the fastest speed profiles (flat out) due to the additional mass of the storage device.

\section{References}

[1] Khmelnitsky, E., "On an Optimal Control Problem of Train Operation," IEEE Transactions on Automatic Control, vol. 45, pp. 1257, 2000.

[2] Howlett, P., "The Optimal Control of a Train," Annals of Operations Research, vol. 98, pp. 65, 2000.

[3] Franke, R., Terwiesch, P., and Meyer, M., "An algorithm for the optimal control of the driving of trains," Proceedings Of The 39th IEEE Conference On Decision And Control, Vols 1-5, pp. 2123-2128, 2000.

[4] Ko, H., Koseki, T., and Miyatake, M., "Application of dynamic programming to the optimization of the running profile of a train," Computers in Railways IX, vol. 15, pp. 103-112, 2004.

[5] Lukaszewicz, P., "Energy Consumption and Running Time for Trains," in KTH, Department of Vehicle Engineering: Royal Institute of Technology, Stockholm, 2001, pp. 153.

[6] Bocharnikov, Y. V., Tobias, A. M., Roberts, C., Hillmansen, S., and Goodman, C. J., "Optimal driving strategy for traction energy saving on DC suburban railways," IET Electric Power Applications, vol. 1, pp. 675, 2007.

[7] Chuang, H. J., Chen, C. S., Lin, C. H., Hsieh, C. H., and Ho, C. Y., "Design of optimal coasting speed for saving social cost in mass rapid transit systems," 2008 Third International Conference On Electric Utility 
Deregulation And Restructuring And Power Technologies, Vols 1-6, pp. 2833-2839, 2008.

[8] Chang, C. S., Xu, D. Y., and Quek, H. B., "Pareto-optimal set based multiobjective tuning of fuzzy automatic train operation for mass transit system," IEE Proceedings-Electric Power Applications, vol. 146, pp. 577583, 1999.

[9] Domínguez, M., Fernández, A., Cucala, A. P., and Cayuela, L. P., "Computer-aided design of ATO speed commands according to energy consumption criteria," Computers In Railways XI - Computer System Design And Operation In The Railway And Other Transit Systems, vol. 103, pp. 183-192, 2008.

[10] Ramos, A., Peña, M. T., Fernández-Cardador, A., and Cucala, A. P., "Mathematical programming approach to underground timetabling problem for maximizing time synchronization," Revista CEPADE, pp. 95, 2008.

[11] Sagareli, S. and Gelman, V., "Implementation of new technologies in traction power systems," presented at Proceedings of the 2004 ASME/IEEE Joint Rail Conference (IEEE Cat. No.04CH-37550),

[12] Siemens, "SITRAS ${ }^{\circ}$ SES Acumulador de energía para el transporte de cercanías."

[13] Gunselmann, W., "Technologies for Increased Energy Efficiency in Railway Systems," 2005.

[14] Chymera, M., Renfrew, A., and Barnes, M., "Energy Storage Devices in Railway Systems," IEE, 2006.

[15] M. Miyatake, K. Matsuda, and Haga, H., "Charge/discharge control of a train with on-board energy storage devices for energy minimization and consideration of catenary free operation," presented at Computer in Railways XI, Toledo, Spain, 2008.

[16] Steiner, M. and Scholten, J., "Energy storage on board of DC fed railway vehicles PESC 2004 Conference in Aachen, Germany," Pesc 04: 2004 IEEE 35th Annual Power Electronics Specialists Conference, Vols 1-6, Conference Proceedings, pp. 666-671, 2004.

[17] Steiner, D. M., Klohr, M., and Pagiela, S., "Energy storage system with Ultracaps on board of railway vehicles," 2007 European Conference on Power Electronics and Applications, Vols 1-10, pp. 982-991, 2007.

[18] Gay, S. E. and Ehsani, M., "On-board electrically peaking drive train for electric railway vehicles," presented at 2002 IEEE 56th Vehicular Technology Conference Proceedings (Cat. No.02CH37359) 